\title{
Computing method of ray intercept in radio wave tunnel perspective technology
}

\author{
Xingang $\mathrm{Xu}$, Jianhua Yue, Zhixin Liu \\ School of Resource and Earth Science \\ China University of Mining \& Technology \\ Xuzhou, China \\ xuxingang@126.com
}

\author{
$\mathrm{Hu}$ Wang \\ School of Compute Science and Technology \\ China University of Mining \& Technology \\ Xuzhou, China
}

\begin{abstract}
Ray intercept algorithm is the key factor to improve the speed of radio tomography. A second scan search algorithm is proposed to acquire the intersecting point coordinates between ray and gridlines. For this algorithm, successive scanning and sorting of intersection sequence would consume much computing time and memory. In order to avoid successive scanning, search variables have been introduced into search procedure to reduced scan range. Search progress has been improved to omit the sort process at the same time. Through these two improvements, the number of searches has been greatly reduced. The algorithm proposed in this paper is proved to be effective and accurate by electromagnetic wave amplitude tomography inversion calculation of a certain coal face fault structure perspective data.
\end{abstract}

Keywords-radio wave tunnel perspective; computed tomography; ray intercept; search variables

\section{INTRODUCTION}

Radio wave tunnel perspective method serves as a geophysical method using electromagnetic wave in coal seam waveguide transmission characteristics, through which geological structure in coal seam can be researched ${ }^{[1]}$. This technology was put forward to detect blind ore bodies by the former Soviet union scientists's Petrovsky in $1923^{[2]}$. A similar study has been carried out in the United States, the Czech republic, Bulgaria and so on ${ }^{[3-6]}$. This work was started in china from the late $1950 \mathrm{~s}^{[7-12]}$.

Radio wave tunnel perspective method is different from other electromagnetic perspective method because only a few tunnel can be utilized to work. The data of radio wave tunnel perspective method is incomplete for a few observation direction and big distance of measuring points, so the inversion results is not unique. Increasing the density of acquisition is beneficial to enhance precision. Tomography inversion of high dense acquisition's data can cause a huge amount of calculation. Intercept calculation will consume much time and memory in this progress. Concise and efficient ray intercept algorithm is good for developing high resolution electromagnetic wave tomography, but less research work has been done in this field so far. The classic computing intercept way was given by Siddon in $1985^{[13]}$. Some scholars have improved this algorithm, Such as Zhang Shunli ${ }^{[14]}$. Siddon algorithm and improved algorithms are applied to all azimuth observation data in medical CT, which are complicated and slow for radio wave tunnel perspective method. Radio wave tunnels perspective tomography is a two-dimensional reconstruction process. In this paper, a more simple and efficient algorithm is researched for radio wave tunnels perspective tomography.

\section{ALGORITHM PRINCIPLE}

In order to detect structure within the coal working face, radio transmitters are put in a location of A roadway and the electromagnetic wave is continuous launched in a certain period of time. At the same time, radio receivers are placed in $B$ roadway and moved for measuring magnetic field amplitude value. There are usually 10 to 12 radio receivers position associated with a launch. In order to detect the face within the structure, a few launch positions are designed in two roadway. The set of transmitter and receiver locations and the Shot line path are shown in Fig.1.

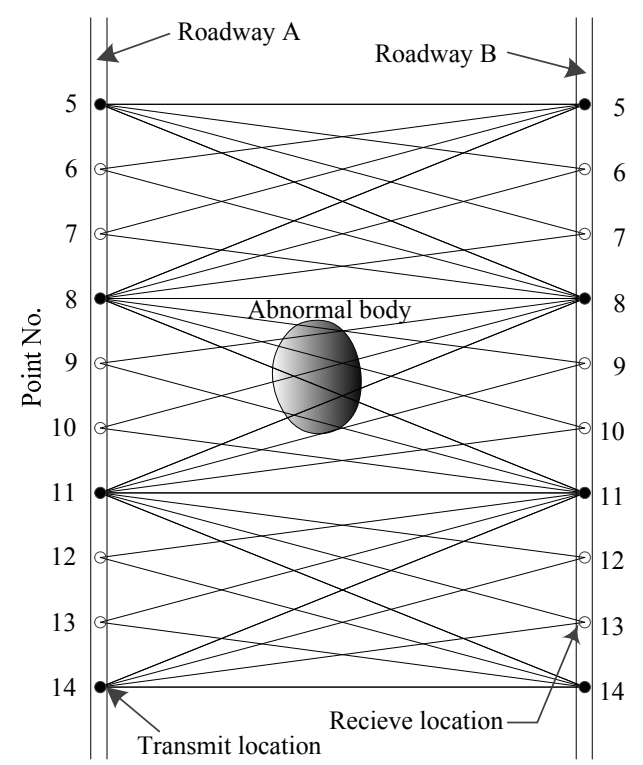

Fig. 1. The set of transmitter and receiver locations (and the Shot line path) used for detecting geologic structure in mine face. 
Dipole antenna is used in radio wave tunnels perspective exploration. Magnetic field strength $\mathrm{H}$ at the receiver can be expressed as

$$
H=H_{0} \frac{e^{-\beta r}}{r}
$$

where $\mathrm{H}_{0}$ is initial magnetic field strength at the launcher, $\beta$ is absorption coefficient, $r$ is the distance between the launcher and the receiver, $\theta$ is angle between transmit antenna axis and the receiving direction. For radio wave tunnels perspective, $\sin \theta$ usually equal to 1 . Eq.(1) may be written as

$$
\beta r=H_{0}-H-10 \lg r
$$

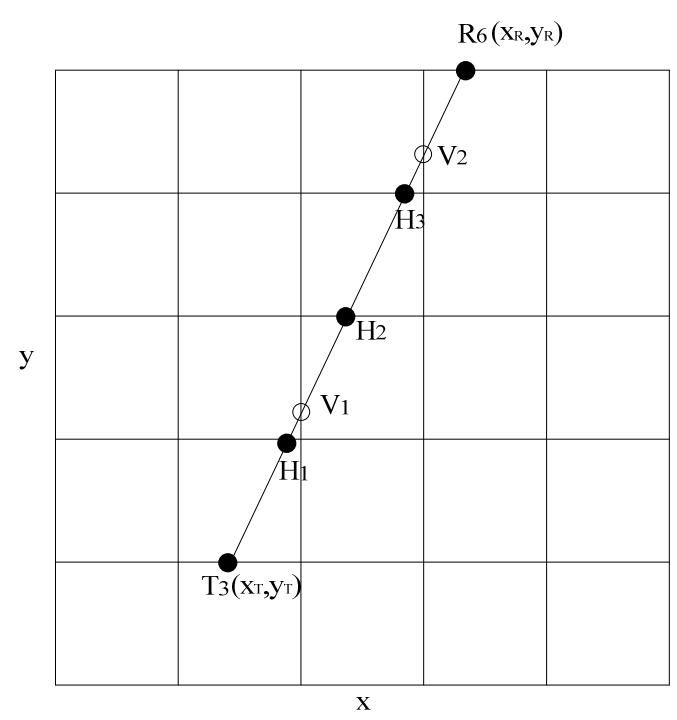

Fig. 2. Two dimensional rectangular domain with a uniform square mesh in coal face.

Coal mining face is divided into equidistant rectangle ,as shown in Fig.2. The number of nodes in the horizontal direction is $\mathrm{N}_{\mathrm{x}}$, and the number of nodes in the vertical direction is $\mathrm{N}_{\mathrm{y}}$. The coordinate of the launcher is $\left(\mathrm{x}_{\mathrm{T}}, \mathrm{y}_{\mathrm{T}}\right)$, The coordinate of the launcher is $\left(\mathrm{x}_{\mathrm{R}}, \mathrm{y}_{\mathrm{R}}\right)$. $\Delta d$ is mesh spacing.

Denoting a particular mesh unit absorption coefficient as $\beta_{j}$, the length of straight-line path contained by that mesh unit as $d_{i j}$, and that length of ray path is $r_{i}$, the path may be written as

$$
\sum_{j=1}^{n} d_{i j} \beta_{j}=H_{0}-H_{i}-10 \lg r_{i}
$$

where $i$ is sequence number of ray, $j$ is sequence number of mesh.

Let $s_{i}=H_{0}-H_{i}-10 \lg r_{i}$, then Eq.(3) may be written as

$$
\sum_{j=1}^{n} d_{i j} \beta_{j}=s_{i}
$$

For Eq.(4), One solution method is iterative method. Ray intercept $d_{i j}$ is important in the process of solving. The primary task is computing coordinate of intersection point between ray and gridline in the process of acquiring ray intercept $d_{i j}$.

The straight line from point $T_{3}$ to point $R_{6}$ may be represented parametrically as

$$
y=\frac{y_{\mathrm{T}}-y_{\mathrm{R}}}{x_{\mathrm{T}}-x_{\mathrm{R}}} x+\frac{y_{\mathrm{R}} x_{\mathrm{T}}-y_{\mathrm{T}} x_{\mathrm{R}}}{x_{\mathrm{T}}-x_{\mathrm{R}}}
$$

Coordinates of intersection points between ray and gridline may be obtained by second scan search algorithm. It requires the following steps.

- If the $x_{\mathrm{T}}=x_{\mathrm{R}}$, coordinate $\mathrm{x}$ of intersection point is equal to $x_{\mathrm{T}}$. Coordinate $\mathrm{y}$ of intersection point is equal to coordinate $y_{i}$ of each horizontal gridline.

- If the $x_{\mathrm{T}} \neq x_{\mathrm{R}}$, coordinate $\mathrm{x}$ of intersection point between ray and vertical gridline may be firstly obtained. Coordinate y can be get from Eq.(5). These coordinates are stored in a sequence.

- In the same way to calculate the coordinates of intersection point between ray and horizontal gridline, and store in another sequence.

The intersection point' coordinates obtained by the above algorithm are alone in the two sequences. For acquiring ray intercept $d i j$, they must be merged in a sequence. The merged sequence is not satisfied with the only and adjacent principle. To the requirement of uniqueness a criterion can be added to the second search process: if meeting the $\operatorname{MOD}\left(x_{\mathrm{i}}, \Delta d\right)=0 \& \&$ $\operatorname{MOD}\left(y_{\mathrm{i}}, \Delta d\right)=0$, this intersection point's coordinate is not saved. In order to meet the requirements of the adjacent, the coordinates in merged sequence must be sorted.

\section{IMPROVED METHOD}

\section{A. Introducing Search Variables}

The number of gridlines is very large, but only few intersect ray. If some gridlines close to ray involve judgement alone, the search efficiency may be greatly improved. For vertical direction searching, variables $N_{\text {x-first }}$ and $N_{\text {x-end }}$ are given by the following:

$$
\begin{aligned}
& N_{\text {x-first }}=\frac{\min \left\{x_{T}, x_{R}\right\}}{\Delta d} \\
& N_{\text {x-end }}=\frac{\max \left\{x_{T}, x_{R}\right\}}{\Delta d}+1
\end{aligned}
$$

where $N_{\mathrm{x} \text {-first }}$ is the initial number of node, and $N_{\mathrm{x} \text {-end }}$ is the last number of node. The search scope range from $N_{\mathrm{x} \text {-first }}$ to $N_{\mathrm{x}-}$ end. The $\mathrm{x}$ coordinate values can be given by

$$
x_{i}=i \times \Delta d, \quad i=N_{\mathrm{x}-\text { first }}, \ldots, N_{\mathrm{x} \text {-end }}
$$

The $\mathrm{y}_{\mathrm{i}}$ coordinate value can be gained from Eq.(5). In the same way, the variables $N_{\text {y-first }}$ and $N_{\text {y-end }}$ are defined for horizontal direction searching.

\section{B. Improving search progress}

For the above algorithm, the search process includes horizontal direction and vertical direction alone. The computing time and memory is wasted by the merged sequence sort. Here, we improve this step.

Angle between ray and roadway is lesser than $45^{\circ}$ for radio wave tunnels perspective, so that the slope $\mathrm{k}$ value of ray is greater than 1 or lesser than -1 . The case of $k>1$ and $k<-1$ is symmetrical, therefore, only $\mathrm{k}>1$ condition is discussed. 


$$
\text { If } \mathrm{k}>1, \frac{\left|x_{\mathrm{T}}-x_{\mathrm{R}}\right|}{\Delta d}<\frac{\left|y_{\mathrm{T}}-y_{\mathrm{R}}\right|}{\Delta d}
$$

It is shown from Eq.(8) that the intersections' number of ray and vertical gridlines is lesser than the intersections' number of ray and horizontal gridlines. Firstly, the intersection of ray and horizontal gridlines are sought from $N_{\text {y-first }}$ to $N_{\text {y-end. }}$. There are the following relations among ray, vertical gridlines and horizontal gridlines:

Assuming that the adjacent three intersections $\mathrm{J}_{1}, \mathrm{~J}_{2}, \mathrm{~J}_{3}$, coordinates respectively are $\left(\mathrm{x}_{\mathrm{n}-1}, \mathrm{y}_{\mathrm{n}-1}\right),\left(\mathrm{x}_{\mathrm{n}}, \mathrm{y}_{\mathrm{n}}\right),\left(\mathrm{x}_{\mathrm{n}+1}, \mathrm{y}_{\mathrm{n}+1}\right) . \mathrm{J}_{1}, \mathrm{~J}_{3}$ intersect horizontal gridlines, and $\mathrm{J}_{2}$ intersects vertical gridlines. There is the following relationship:

$$
\left\lfloor\frac{x_{n-1}}{\Delta d}\right\rfloor-\left\lfloor\frac{x_{n+1}}{\Delta d}\right\rfloor=1
$$

Adjacent intersections $\mathrm{J}_{1}$ and $\mathrm{J}_{3}$ have been known. If the right side of Eq.(9) is equal to 1, there is a intersection $\mathrm{J}_{2}$.Then, $\mathrm{J}_{2}$ coordinate may be computed and inserted_at position between $J_{1}$ and $J_{2}$ in the sequence. If the right side of Eq.(9) is equal to 0 , there is no intersection.

This improvement can give rise to decreasing one search and omitting the sort so that the computing time and memory is saved.

\section{Effect Analysis of Improvement}

Assuming that there is coal mining face, the inclined length of working face is 150 meters, and strike length of working face is 1000 meters. The face is divided into equidistant rectangle with 1 meter interval. Receiver spacing is 10 meters, and launcher spacing is 50 meters. There are fifteen receiver along with one launcher. Ray may be controlled in a $70 \mathrm{~m} \times 150 \mathrm{~m}$ area. For every ray, there are 1152 searches if search variables are not used, another only 222 searches with search variables used.585900 searches are reduced for all launchers. In addition, 95130 vertical searches ,sorting and rechecking are saved by the search process improvements.

\section{EXAMPLES}

In order to verify the validity of the algorithm, actual data measured by radio wave perspective method is computed. Geological condition of coal mining working face is shown in Fig. 3. Six faults and one Coal seam thinning belt are disclosed in roadway construction process. Transmitting antenna center frequency is $0.3 \mathrm{MHz}$. The fixed point method is used. The spacings of launchers and receivers are 50 meters and 10 meters, respectively. Amplitude-attenuated imaging results are calculated, as shown in Fig. 4. Extension direction and scope of simple geological structure within the coal working face has been well described, such as fault F1,F2 and thinning belt. One of complex geological structure, such as fault F3, F4, F5, F6, are difficult to distinguish. Improved intercept algorithm in this paper is proved to be correct and efficient.

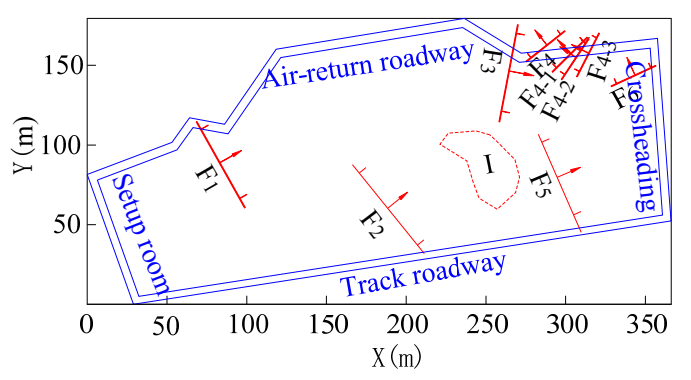

Fig. 3. General situation of coal mining face. Main geological structures have been revealed by tunneling and drilling. There is complex fault zone at the crossing of Air-return roadway and crossheading.

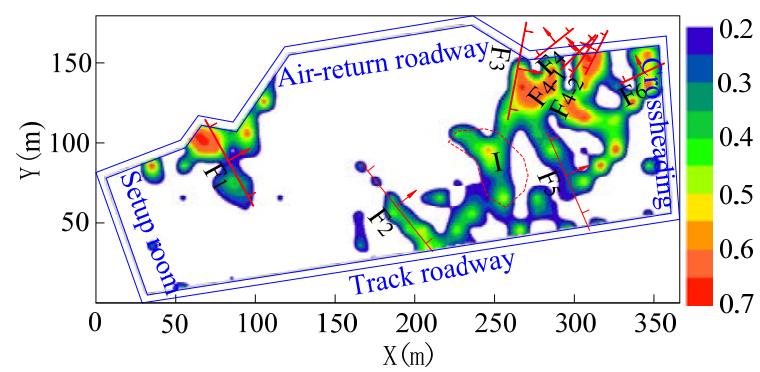

Fig. 4. A pictorial representation of the reconstruction from measured data in coal mining face. Absorption coefficient is larger in red area. It means that coal seam has been destroyed in this area.

\section{CONCLUSION}

The characteristics of the only and the adjacent in intersection point sequence of ray and grid lines have been discussed. On this basis, the search variables have been introduced. Whether ray and gridline intersect or not only is decided within the scope of transmitter and receiver coordinates. As a result, the searching scope is reduced so that Search efficiency is improved. In addition, the further improved search process give rise to decreasing one search and omitting the sort. This computing method of ray intercept is fit for two-dimensional reconstruction process of radio wave tunnels perspective.

\section{ACKNOWLEDGMENT}

Thanks are due to Yang Haiyang for valuable discussion and to $\mathrm{Lu}$ Ran for assistance with engineering data acquisitions.

\section{REFERENCES}

[1] Liu T F, Li Z D, Mine geophysics(in Chinese) ,1st ed. Beijing, China :Coal Industry Press,1993,pp.17-18.

[2] Petrovsky A D, Underground radio wave method(in Chinese) ,1st ed. Translated by Lu K, Cai G M. Beijing, China: Geological Publishing House, 1981,pp.1-2.

[3] Lager D L, Lytle R J, "Determining a subsurface electromagnetic profile from high-frequency measurements by applying reconstructiontechnique algorithms," Radio sei, vol. 12,no. 2, pp.249-260, Mar-Apr. 1977.

[4] Stolarczyk L, Peng S, Luo Y, "Imaging Ahead of Mining with Radio Imaging Method (RIM-IV) Instrumentation and Three-Dimensional Tomography Software," in $22^{\text {nd }}$ International Conference on Ground Control in Mining,2003, pp.136-147. 
[5] Ernst J R, Green A G, Maurer H, Holliger K, "Application of a new 2D time-domain full-waveform inversion scheme to crosshole radar data," Geophysics, vol. 72, no. 5, pp.53-56, Sep-Oct. 2007.

[6] Ernst J R, Maurer H, Green A G, Holliger K, "Full-waveform inversion of crosshole radar data based on 2-D finite-difference time-domain solutions of Maxwell's equations," IEEE Trans. Geosci. Remote Sens., vol. 45, no. 9, pp.2807-2828, Sep. 2007.

[7] Liu L Z, "New progress of electromagnetic wave method in well," Oil and gas prospecting abroad(in Chinese), vol. 6, no. 6, pp.747-762, Nov. 1994.

[8] Dong S H, Wang Q, "Application of tomography in radio wave tunnels perspective," Journal of China University of Mining \& Technology(in Chinese), vol. 32, no. 5, pp. 579-582,Sep. 2003.

[9] Liu X M, Liu S C, Jiang Z H, "Study on propagation attenuation features of random incidence angle electromagnetic wave in lossy medium ," Coal Science and Technology(in Chinese), vol. 40, no. 6, pp.96-99, 103, Jun. 2012.
[10] Liu X M, Liu S C, Jiang Z H, "Study on the tomography of radio-wave penetration based on improved amplitude attenuation constant," Progress in Geophysics(in Chinese), vol. 28, no. 2, pp.980-987,Apr. 2013.

[11] Liu Z X, Liu S C, Wang D W, "Phase tomography technology of tunnel radio wave perspective," The Chinese Journal of Nonferrous Metals(in Chinese), vol. 23, no. 9, pp. 2371-2378, Sep. 2013.

[12] Ning S N, Zhang S H, Yang F, "Radio wave tomography technique and its application in underground radio wave probing," Journal of China Coal Society(in Chinese), vol. 26, no. 5, pp. 468-472,Oct. 2001.

[13] Siddon R L, "Fast calculation of the exact radiological path for a threedimensional CT array," Med Phys, vol. 12, no. 2, pp. 252-255,Mar-Apr. 1985.

[14] Zhang S L, Zhang D H, Li S, "Research of Fast Image Reconstruction on ART Algorithm," Computer Engineering and Applications(in Chinese), vol.42, no. 24, pp.1-3, Aug. 2006. 